\title{
Control of multi-terminal HVDC networks towards wind power integration: A review
}

\author{
Fernando D. Bianchi ${ }^{\mathrm{a}}$, José Luis Domínguez-García ${ }^{\mathrm{a}}$, Oriol Gomis-Bellmunt ${ }^{\mathrm{a}, \mathrm{b}}$ \\ ${ }^{a}$ IREC Catalonia Institute for Energy Research, Jardins de les Dones de Negre 1, $2 a$. \\ 08930 Sant Adrià de Besòs, Barcelona, Spain \\ ${ }^{b}$ Centre d'Innovació Tecnològica en Convertidors Estàtics i Accionaments (CITCEA-UPC), \\ Universitat Politècnica de Catalunya UPC, Av. Diagonal, 647, Pl. 2. 08028 Barcelona, \\ Spain
}

\begin{abstract}
More interconnections among countries and synchronous areas are foreseen in order to fulfil the EU 2050 Target on the renewable generation share. One proposal to accomplish this challenging objective is the development of the socalled European SuperGrid. Multi-terminal HVDC networks are emerging as the most promising technologies to develop such a concept. Moreover, multiterminal HVDC grids are based on highly controllable devices, which may allow not only transmitting power, but also supporting the $\mathrm{AC}$ grids to ensure a secure and stable operation. This article aims to present an overview of different control schemes for multi-terminal HVDC grids, including the control of the power converters and the controls for power sharing and the provision of ancillary services. The article also analyses the proposed modifications of the existing control schemes to manage high participation shares of wind power generation in multi-terminal grids.
\end{abstract}

Keywords: Multi-terminal HVDC; ancillary services; distributed control; wind power integration; power sharing methods; voltage source converters.

\section{Introduction}

European Union (EU) has defined as a target for 2050 to reduce about 8095\% the greenhouse gas emissions compared to 1990 levels. This implies that the penetration of renewable energy sources in power systems should increase up to $100 \%$ [1]. Under this scenario, the European Wind Initiative (EWI) has established as strategic objective to enable wind energy to supply half of Europe's electricity for 2050 [2].

Currently, wind power is the generating technology with the highest rate of new installations in Europe, according to EWEA [3]. In 2014, the new installations of wind power plants (WPPs) accounted for $43.7 \%$ of total new installed

Email address: fbianchi@irec.cat Corresponding author (Fernando D. Bianchi) 
capacity. Moreover, the share of wind energy in the EU power generation mix has increased from 2.4\% in 2000 to $14.1 \%$ in 2014. From the total EU electricity consumption of $2798 \mathrm{TWh}, 9.1 \%$ is provided by onshore WPPs and $1.1 \%$ by offshore plants [3]. Offshore locations are gaining presence on wind sector as a consequence of the limited available inland sites to install new wind farms (especially in Europe). These locations also offer better wind conditions, less turbulence and higher mean wind speeds.

To fulfil the EU 2050 Target, large interconnections among different countries as well as different synchronous areas in Europe will be necessary in order to share renewable power generation with other systems (e.g. wind power from the North Sea, hydro power from Norway or PV power from the southern countries). The development of an offshore grid using existing and planned offshore installations may help to achieve this target. An alternative to reach such an objective is the so-called SuperGrid [4]. A SuperGrid can be defined as an overlay system interconnecting different transmission networks. This new grid is expected to be implemented with Multi-Terminal HVDC (MT-HVDC) networks based on voltage source converter (VSC) technologies [5]. The main benefits of using VSC are the capability to control active and reactive power independently, the capacity of establishing an independent asynchronous AC grid without additional devices, and the reduction of the output filters needed for low harmonic distortion. Nowadays, MT-HVDC grids are emerging as a promising concept and are rapidly attracting interest and effort from both academia and industry. This trend is clear in the specific programmes of international entities (e.g. CIGRÉ working groups B4-56 to B4-60 and Friends of SuperGrid), in EU funded projects (e.g. TWENTIES, MEDOW, BESTPATH and IRPWIND) and in the commissioning of the first 5-terminal HVDC network in China in early 2014 [6].

The wide use of MT-HVDC systems also raises a number of challenges. One of the key technological challenges is the operation and control of MTHVDC transmission systems [7]. Another challenge is to deal with the reduction of the global inertia and the changes in the dynamics of conventional power systems, caused by the continuous increase of both renewable generation and MT-HVDC systems. For that reason, transmission system operators (TSOs) are developing new grid codes with specific requirements for HVDC systems aimed at ensuring a proper network operation, including frequency support, power oscillation damping and inertia emulation [8].

This article presents a review of the main contributions addressing both the operation and control of MT-HVDC grids and their interactions with the AC networks. Different control levels are reviewed, including the low-level control in VSCs. The control schemes are organised in two sections according to the main objectives: power sharing and provision of ancillary services. The article also analyses the proposed modifications of the existing control schemes to manage the wind power integration in MT-HVDC grids. 


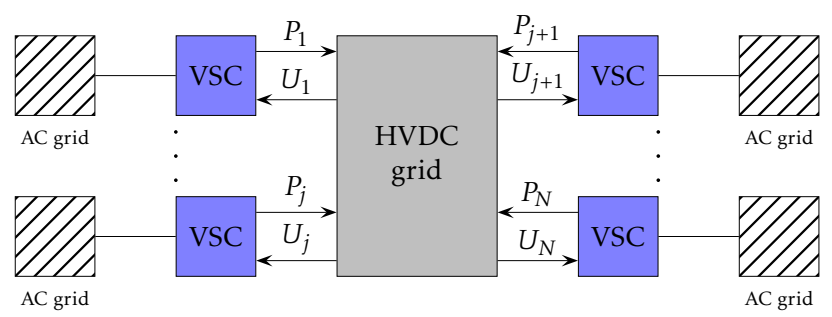

Figure 1: Representation of a generic MT-HVDC network

\section{Hierarchical control structure in MT-HVDC networks}

A generic representation of a MT-HVDC network consisting of $N$ stations is sketched in Figure 1. At each terminal, a VSC connects the HVDC grid with an AC grid. Some of these grids inject power into the multi-terminal grid and others extract power. The first group includes for instance WPPs and the second main shore networks.

The main purpose of a multi-terminal network is to share power among the AC grids. To fulfil this objective, one or more VSC must regulate the DC voltage at every terminal $j$. Since MT-HVDC networks are systems based on power electronics (which provide high controllability), there is also an increasing interest in MT-HVDC networks providing additional services for improving stability of main AC grids; such as frequency support, damping power oscillations, among others.

In MT-HVDC networks, the power sharing is achieved by regulating the DC voltage at all terminals. Therefore, at least one of the VSC connected to the multi-terminal grid must regulate the DC voltage. However, additional controls are also necessary to ensure that the desired power values are transferred to their respective terminals with the best efficiency. In addition, the system must continue working under certain faults and abnormal conditions. Similar to AC systems, several control levels are needed to fulfil these objectives.

Figure 2 shows the four-level hierarchy control scheme proposed in [9]. The current control is the lowest level and is responsible for regulating the current injected by each power converter (VSC). The primary control level aims to regulate the $\mathrm{DC}$ voltage using local information (DC voltage and power values at terminals) and imposes the set-points to the current control loop. The primary level is implemented with PI or droop controllers in the VSCs responsible for regulating the voltage. This control must be decentralised in order to ensure a suitable behaviour even under faults or other abnormal conditions, e.g. faults in communications. The secondary level produces the voltage set-points for the primary controls with the aim of restoring the power flow after a contingency and thus ensuring the desired power exchange among terminals. This control is slower than the primary control and can be centralised or distributed, using communication channels to fulfil its goal. The tertiary level is focussed on long term planning. For instance, in [10-12], an optimisation algorithm is proposed 


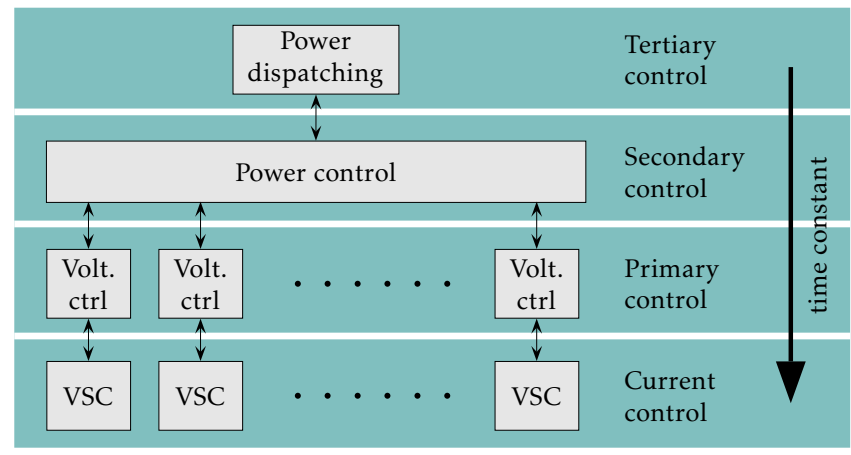

Figure 2: Hierarchical control structure for MT-HVDC networks

to generate voltage set-points to slack buses with the aim of minimising the system losses. With a similar objective, Aragüés-Peñalba et al. [13] present an optimal tertiary control for a MT-HVDC network based on droop control. In this case, the tertiary control produces the set-points for the power control.

All control levels participate more or less in the power sharing and in the provision of ancillary services depending on the particular control scheme. However, the primary control is mainly aimed at guaranteeing a suitable power sharing as this level is capable of working without communications. This ensures a suitable power sharing with the aim of maintaining stable the main electrical variables even under abnormal conditions. The other levels in general contribute to a more efficient operation of the entire system. The provision of ancillary services is mainly implemented in the secondary control, although there are some examples in which the primary control also participates in the fulfilment of this objective. Before describing the different control schemes for power sharing and the provision of ancillary services, a brief review of the control of VSC in the context of MT-HVDC network applications is presented as these are key parts in all control schemes.

\section{Control of VSCs for MT-HVDC networks}

The most common control strategy for VSCs relies on Park transformations and PI controllers. The idea is to project the $a b c$ variables into the voltage oriented synchronous reference $d q$ frame. Thus, the design can be stated as the tuning of several PI controllers seeking to regulate DC variables. This control scheme consists of two loops and is sketched in Figure 3. The inner one focusses on regulating the converter currents and the outer loop aims to fulfil other objectives as voltage or power regulation. In this scheme, balanced voltages are assumed.

\subsection{Current control}

As shown in Figure 3, the AC voltages and currents in the $a b c$ frame are referred to the $d q$ frame using the Park transformation. After this, the dynamics 


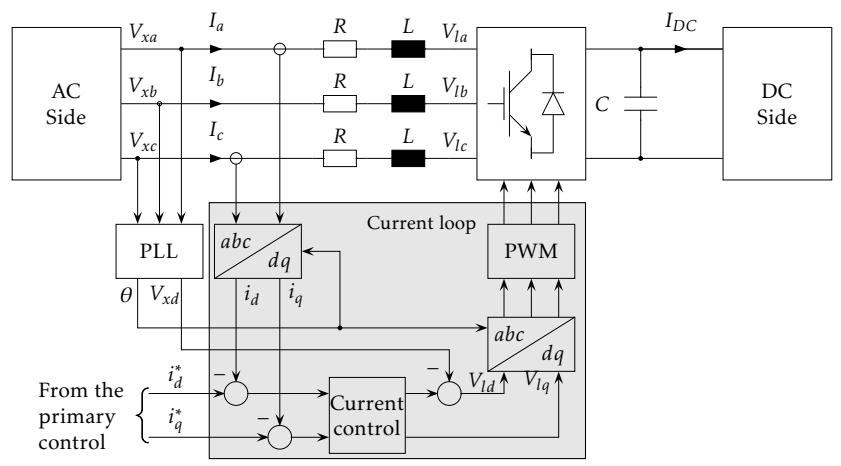

Figure 3: Common control scheme for VSCs

of the AC currents $I_{a b c}$ is given by

$$
\begin{aligned}
\frac{d i_{d}}{d t} & =-\frac{R}{L} i_{d}+\omega i_{q}+\frac{1}{L}\left(V_{x d}-V_{l d}\right), \\
\frac{d i_{q}}{d t} & =-\frac{R}{L} i_{q}+\omega i_{d}-\frac{1}{L} V_{l q},
\end{aligned}
$$

where $\omega$ is the frequency, $i_{d}$ and $i_{q}$ are the direct and quadrature currents through the $L R$ filter, $V_{l d}$ and $V_{l q}$ are the direct and quadrature voltages generated by the switching devices commanded by the PWM (pulse-width modulation) algorithm and $V_{x d}$ is the direct $\mathrm{AC}$ grid voltage ( $V_{x q}$ is 0 as the $d q$ frame is oriented to the AC grid voltages).

Then, using the control law

$$
\begin{aligned}
& V_{l d}=k_{p c}\left(i_{d}^{*}-i_{d}\right)+k_{i c} \int\left(i_{d}^{*}-i_{d}\right) d t-\omega L i_{q}, \\
& V_{l q}=\underbrace{k_{p c}\left(i_{q}^{*}-i_{q}\right)+k_{i c} \int\left(i_{q}^{*}-i_{q}\right) d t}_{\text {PI control }} \underbrace{-\omega L i_{d}}_{\begin{array}{c}
\text { decoupling } \\
\text { terms }
\end{array}},
\end{aligned}
$$

with $k_{p c}=L / \tau$ and $k_{i c}=R / \tau$, the dynamics of the closed inner loop is given by

$$
\frac{d i_{d}}{d t}=-\frac{1}{\tau}\left(i_{d}-i_{d}^{*}\right), \quad \frac{d i_{q}}{d t}=-\frac{1}{\tau}\left(i_{q}-i_{q}^{*}\right),
$$

where $i_{d}^{*}$ and $i_{q}^{*}$ are the direct and quadrature current set-points. These variables are imposed by the primary control level in order to fulfil several objectives. In this frame, the active and reactive powers are

$$
P=\frac{3}{2} V_{x d} i_{d}, \quad Q=\frac{3}{2} V_{x d} i_{q},
$$

respectively. Therefore, each power can be independently controlled with proper set-points $i_{d}^{*}$ and $i_{q}^{*}$. 
Neglecting the converter losses, the power on the AC side and the DC side coincides, then the DC current is given by

$$
I=\frac{3}{2} \frac{V_{x d} i_{d}}{U},
$$

where $U$ is the $\mathrm{DC}$ voltage with dynamics governed by

$$
\frac{d U}{d t}=\frac{1}{C}\left(I-I_{D C}\right)
$$

with $C$ the filter capacitance and $I_{D C}$ the current injected into the DC grid.

Considering the converter limits, the DC variables must remain in the gray areas illustrated in the U-P and U-I planes in Figure 4. The boundaries of these areas are established by the following considerations.

- Power limits are imposed by the limits in the converter AC currents as a consequence of the semiconductors rating.

- DC voltage limits, the upper voltage limit is imposed by the insulation of the switching devices, the lower one by modulation limitations of the converter topology.

- DC current limits are imposed by the current rating of the components connected on the DC side.

The primary control must ensure the DC variables remain within these limits. These strategies are presented in the next subsection.

\subsection{Primary control at each terminal}

As mentioned before, the primary control is decentralised, which implies that the global behaviour is governed by the coordination of a set of controllers using only local information (e.g. DC voltage, power at each terminal). This is implemented by the outer loop control in the VSCs. In this subsection, we present the different configurations for this controller focussing on the implementation, leaving the coordination and analysis of the entire MT-HVDC grid behaviour for the next section.

There are basically four configurations to implement the primary control at each terminal: constant power, constant DC current, constant DC voltage and droop control. The first two configurations are applied when the VSC injects power into the DC grid from an AC source. The third and fourth ones are used when the converter is regulating the $\mathrm{DC}$ voltage. The droop control is basically a proportional control law that is capable of distributing the voltage regulation among several stations. The four control configurations are implemented in the outer control loop, which provides the set-points to the inner current loop previously described. 

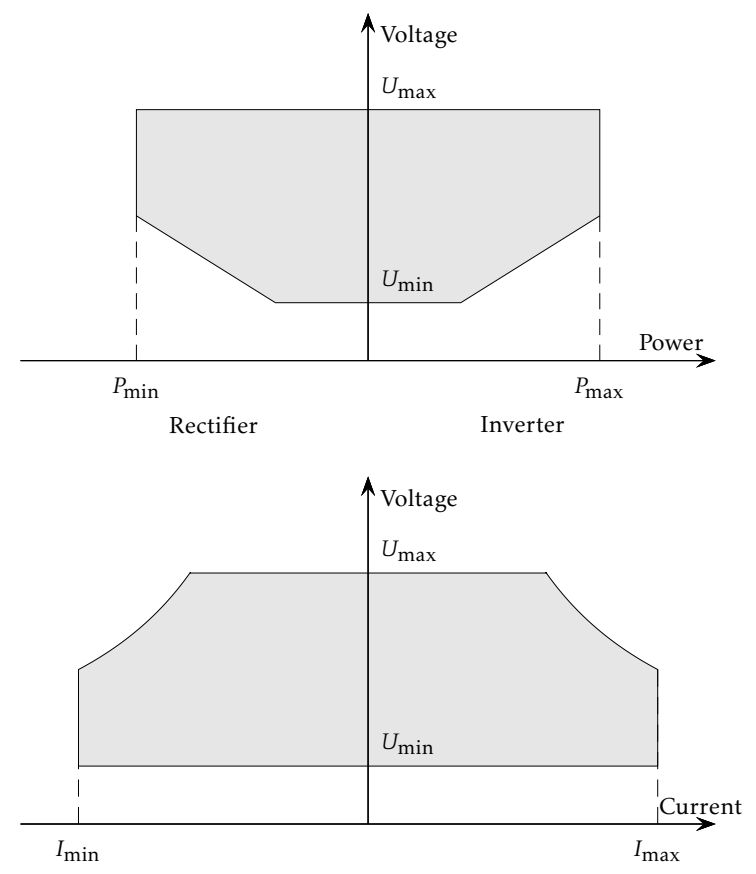

Figure 4: Limits for the DC variables due to converter limits in the U-P and U-I planes

\subsubsection{Constant current control}

In constant current control, the set-point for the direct current $i_{d}$ is given by

$$
i_{d}^{*}=\frac{2 U}{3 V_{x d}} I^{*}
$$

where $I^{*}$ is the desired DC current. This operating configuration is illustrated in the U-P plane in Figure 5 (red line).

\subsubsection{Constant power control}

Constant power control scheme, sketched in Figure 6, includes a PI controller and the factor $2 / 3 V_{x d}$ in order to ensure the power delivered to the DC side is maintained at $P^{*}$. To this end, the current set-point is set as

$$
i_{d}^{*}=\frac{2}{3 V_{x d}} P^{*}
$$

in steady-state. This relationship is obtained from active power calculation on $d q$ frame with $V_{x q}=0$. Figure 5 illustrates the constant power strategy (blue line) in the U-P plane along with the power converter limits. In this case, the VSC controller ensures a constant power without any regulation of the DC voltage. In general, VSC operating in this mode corresponds to power sources that are not responsible for regulating the voltage, such as WPPs. 


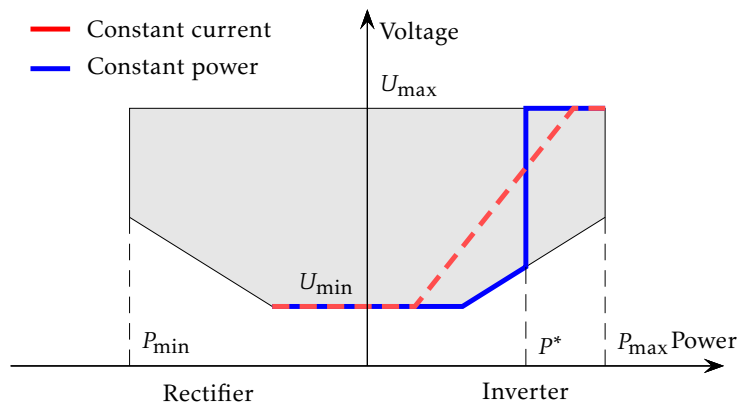

Figure 5: Constant current and constant power strategies in the U-P plane

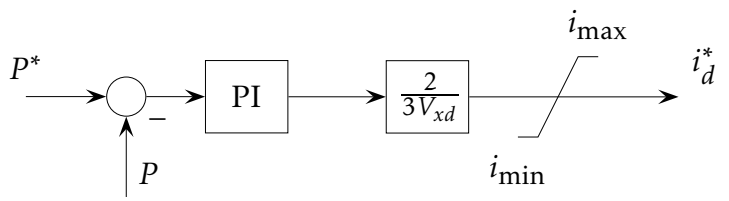

Figure 6: Constant power control scheme

\subsubsection{Constant voltage control}

Figure 7 shows the constant voltage control scheme, which consists of a PI structure and a linearising factor at its output. In order to tune the PI control, consider the power balance

$$
\frac{3 V_{x d} i_{d}}{2}-P-P_{l}-U I_{c a p}=0
$$

where $P$ is the power transferred to the DC grid, $P_{l}$ is the losses and $I_{c a p}$ is the current through the filter capacitor. Then, the $\mathrm{DC}$ voltage dynamics is governed by

$$
\frac{d U}{d t}=\frac{1}{U C}\left(\frac{3 V_{x d} i_{d}}{2}-P-P_{l}\right) .
$$

Assuming the current loop dynamics is faster than the outer loop $\left(i_{d} \approx i_{d}^{*}\right)$ and using a PI controller, the DC voltage control law results

$$
i_{d}^{*}=(\underbrace{k_{p u}\left(U^{*}-U\right)+k_{i u} \int\left(U^{*}-U\right) d t}_{\text {PI control }} \underbrace{U,}_{\substack{\text { linearising } \\ \text { factor }}}
$$

where the controller parameters are given by

$$
k_{p u}=\frac{4 \xi \omega_{n} C}{3 V_{x d}}, \quad \quad k_{i u}=\frac{2 \omega_{n}^{2} C}{3 V_{x d}},
$$

with $\omega_{n}$ and $\xi$ the frequency and damping corresponding to the desired location of the closed-loop poles. 


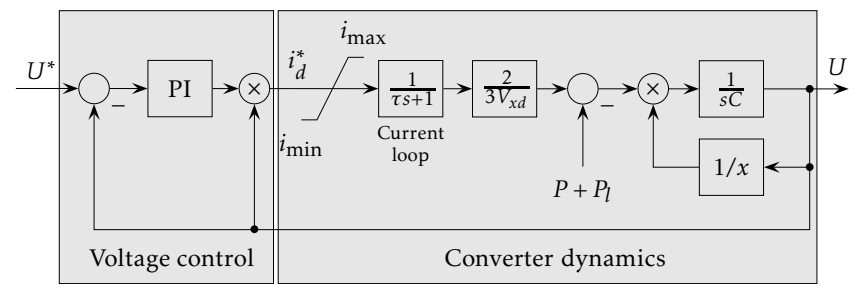

Figure 7: Constant voltage control scheme

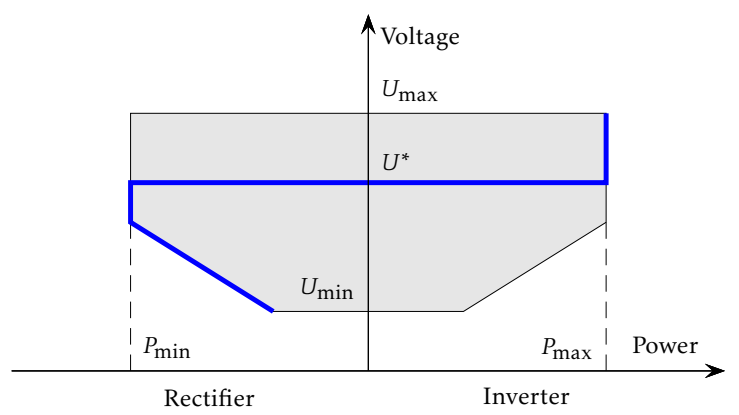

Figure 8: Constant voltage strategy in the U-P plane

The constant voltage control is illustrated in the U-P plane in Figure 8. In this case, the PI controller acts on the power injected/extracted in order to maintain the DC voltage constant at a reference value. Commonly, a converter with this control is called slack bus, since it is responsible for maintaining DC voltage.

\subsubsection{Droop control}

Droop control is basically a proportional control aimed at regulating the DC voltage. The droop control law can act directly on the current

$$
I-I^{*}=K_{I}\left(U-U^{*}\right),
$$

or as input to a constant power control

$$
P-P^{*}=K_{P}\left(U-U^{*}\right) .
$$

This last scheme is illustrated in Figure 9. The droop gains are computed according to the maximum allowed voltage deviations for given current or power changes. The gain values determine the power shared with other converters and also the stability of the entire grid.

Figure 10 shows the current and power droop strategies in the U-P plane. The proportional control allows small deviations from the set-point $U^{*}$ providing flexibility in the power sharing in multi-terminal applications as will be seen in the next sections. 


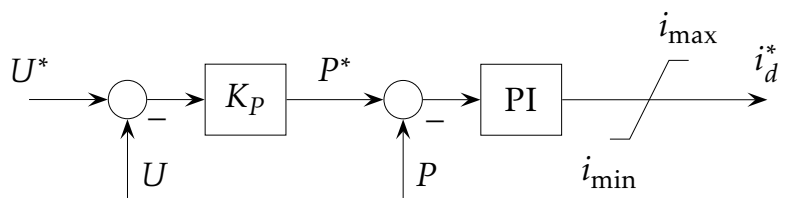

Figure 9: Power droop control scheme

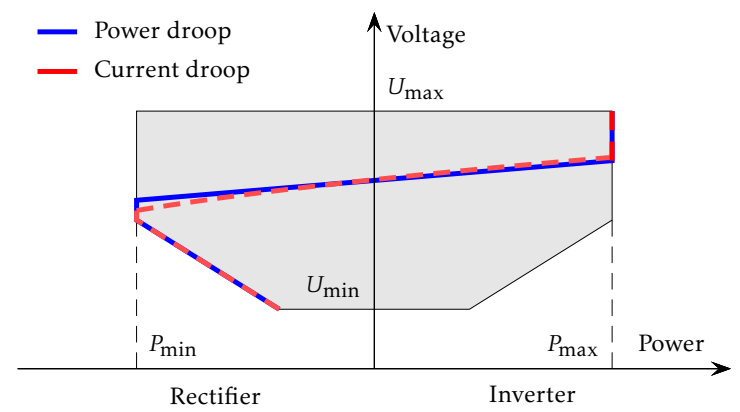

Figure 10: Power and current droop strategies in the U-P plane

\section{Control schemes for power sharing in MT-HVDC networks}

This section analyses the coordination of the primary control schemes presented in Subsection 3.2 in order to ensure a proper power sharing among the stations connected to the MT-HVDC grid. For this aim, at least one of the VSC connected to the multi-terminal grid must regulate the DC voltage. There are several options to fulfil this objective depending on the control strategy at each VSC. In all cases, in order to obtain a feasible operation, only one VSC can operate at constant voltage.

\subsection{Master-slave control}

In master-slave schemes, the DC voltage regulation of the entire network is performed by only one station. To this end, one VSC works in constant voltage mode (master terminal) and the rest of VSCs (slave terminals) are configured in constant power mode. This is illustrated in Figure 11 for a four-terminal network case. In this example, station 1 acts as a master terminal, which is the only responsible for ensuring a proper regulation of the $\mathrm{DC}$ voltages at all terminals of the network. In the U-P plane in Figure 11, the red line corresponds to a constant power characteristic of $\mathrm{VSC}_{1}$. Considering the total power injected by the other terminals $P_{1}$, the system then operates at point $\mathrm{A}$. If the station works properly, the DC voltage is maintained at $U^{*}$ ensuring that power contributions of the other terminals remain within the converter power limits.

With a terminal ensuring tight regulation of DC voltage, a precise control of the power flow can be achieved. The main drawback is the system reliability 

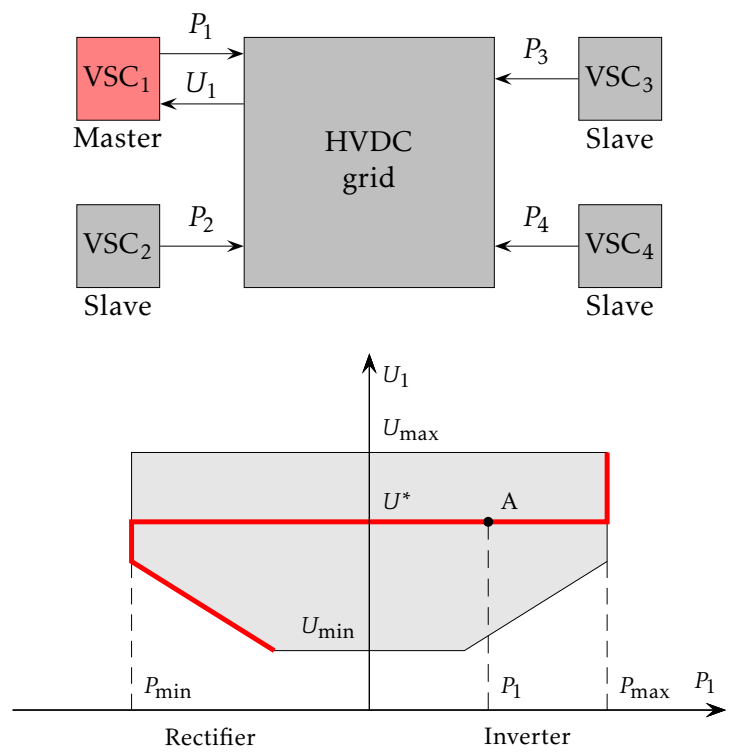

Figure 11: Master-slave control in a four-terminal network. In the upper sketch, the arrows represent information flow

since the control of the entire DC network depends on the proper operation of only one station (the master). In case of outage of the master terminal or $P_{1}$ reaching the converter limits, there would be no control of the DC voltage leading to an outage of the entire DC grid. Furthermore, in order to ensure the system is capable of restoring the DC voltage after any possible imbalance, this scheme requires a large power rating of the master terminal.

As a consequence of these drawbacks, master-slave configurations are not common in MT-HVDC networks, and there are few articles reporting the use of this scheme. In [14], it is analysed a master-slave configuration of a multiterminal with induction generators connected to the slave terminals. Zhang et al. [15] use the master-slave scheme in a micro-grid with wind power, energy storage and loads. These results mainly focus on the distributed generation and the outage of the master-terminal is not analysed. Some simulation results can also be found in [16] where several control methods are compared.

\subsection{Margin voltage control}

The margin voltage control scheme has been proposed to improve reliability by sharing the responsibility for regulating the voltage among two or more terminals $[17,18]$. Basically, this configuration is a master-slave scheme with back-up stations capable of taking over the voltage regulation in case of outage of the master.

The idea is illustrated in Figure 12 for the case of a four-terminal network. The graph at the bottom shows the U-P static characteristics of $\mathrm{VSC}_{1}$ and 


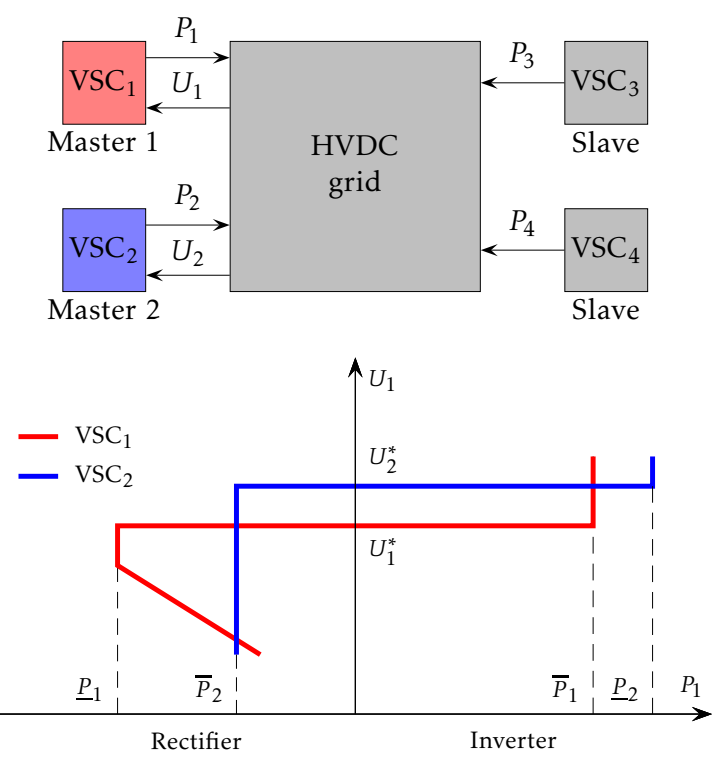

Figure 12: Example of a voltage margin control strategy. In the upper sketch, the arrows represent information flow

$\mathrm{VSC}_{2}$ (referred to station 1 terminals). In normal operation, $\mathrm{VSC}_{1}$ is the master terminal and the rest of the stations are slaves working in constant power mode. Thus, the master station maintains the DC voltage at the set-point $U_{1}^{*}$ and the back-up station $\mathrm{VSC}_{2}$ works in constant power mode with set-point in $\underline{P}_{2}$. In case of an outage of station 1 or reaching its power limits, the DC voltage starts to rise. When the voltage reaches the level $U_{2}^{*}$, the converter in terminal 2 leaves the constant power mode and starts the regulation the DC voltage. In order that this configuration can properly work without communications, the main master station (used in normal operation) must have a lower voltage set-point and the last back-up terminal must be the one with higher voltage set-point.

Figure 13 shows the implementation of the voltage margin control. Normally, the voltage of the entire network is regulated by $\mathrm{VSC}_{1}$ at set-point $U_{1}^{*}$. As $U_{2}^{*}>U_{1}^{*}$, the voltage controller in terminal 2 is limited at the lower level $\bar{P}_{2}$. As a result, station 2 remains working in constant power mode. If the $\mathrm{DC}$ voltage rises, due to an outage of station 1, the PI controller in terminal 2 enters the linear zone and starts to regulate the voltage. The lower power limit in station $\underline{P}_{2}$ can be set by the system operator according to power flow requirements.

As a consequence of the more accurate control of the power flow and the improved reliability, compared to master-slave schemes, this configuration has attracted the attention of several researchers. The proposals include complex schemes with several back-up master stations and DC voltage set-point levels. Nakajima and Irokawa [18] present the application in case of a three-terminal network. In [19], the use of multi-terminal grids with different renewable sources 


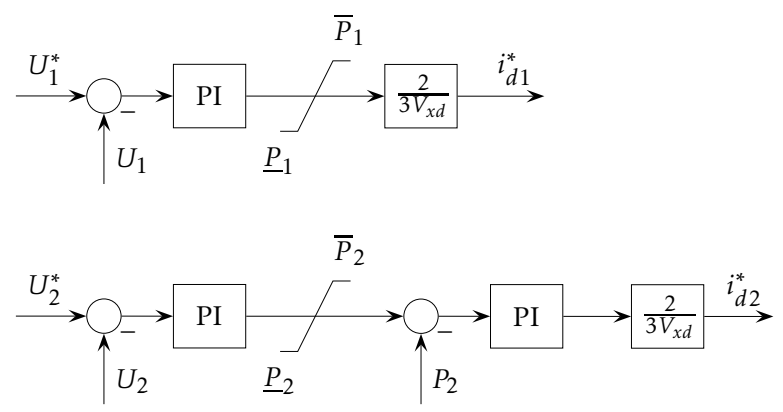

Figure 13: Implementation of voltage margin control

is analysed. Similar results are presented in [20], in case of a multi-terminal with several renewable sources (wind, tidal) and storage. A comparison with other methods is presented in [21]. The operation of this scheme under a converter outage is analysed in [22].

On the downside, the voltage margin control only allows fixed power sharing unless communications are used. To add more flexibility, additional voltage and power set-point can be used, increasing the complexity. Furthermore, the scheme needs several control loops for its implementation which leads to slower voltage regulation [23].

\subsection{Droop control}

The use of droop control in MT-HVDC networks is proposed in [24]. It consists of two or more stations equipped with droop control and the rest of VSCs with constant power control. The idea is similar to the frequency regulation used in AC grids. A power imbalance is reflected as a deviation of the DC voltage, which induces positive or negative contributions from the terminals with droop control. This variation steers the system towards a new stable equilibrium. The operation is illustrated in Figure 14 in the U-P plane. In this case, terminals 1 and 2 regulate the DC voltage and terminals 3 and 4 work at constant power. The system is initially working at point A, where the equilibrium $P_{1 a}+P_{2 a}=P_{3 a}+P_{4 a}$ is reached with voltages $U_{1 a}$ and $U_{2 a}$, respectively. When the total power injected by terminals 3 and 4 rises, the droops at the other terminals lead the system towards the new equilibrium at point $\mathrm{B}$. This causes an increase in the voltage terminals, but also a new power share to accommodate the increase in the total power coming from stations 3 and 4 .

In droop control, the responsibility for the DC voltage regulation is shared among all stations with droop control. As a consequence, in case of an outage of one terminal, the rest of the system is able to continue working. Besides, since all terminals with droop control participate in the voltage and power regulation, large power balances can be managed with stations of small power rating. This high reliability has made the droop scheme the most promising control in MTHVDC networks. The evaluation of this scheme can be found in [16, 22, 25], 

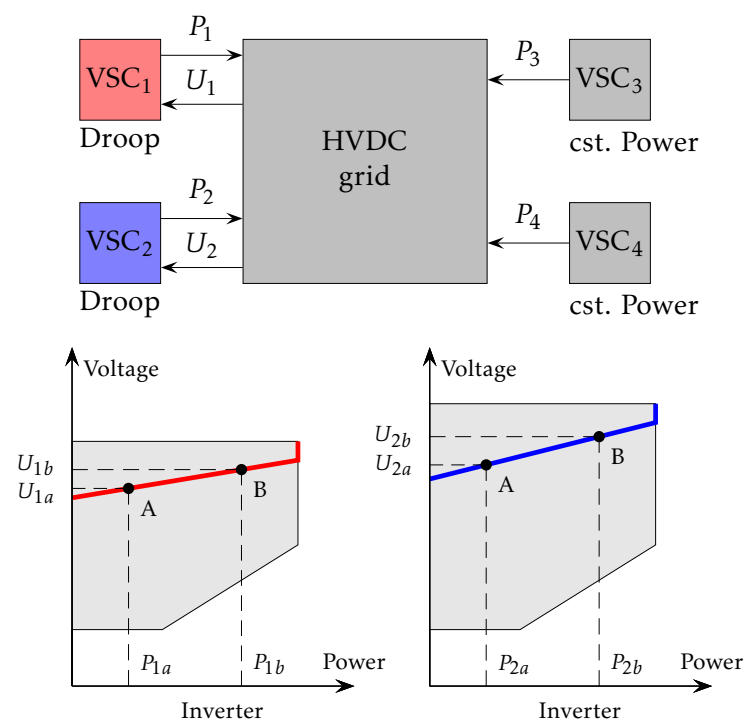

Figure 14: Example of a droop control strategy. In the upper sketch, the arrows represent information flow

among other references. In particular, an experimental implementation in a scale multi-terminal grid is presented in [26].

The main drawback of droop control schemes is the difficulties to ensure a desired power flow as the sharing is governed by the DC voltage errors at each terminal [27]. Sometimes communications are needed for achieving a proper power flow [28]. Besides, large droop gains can provoke oscillations in the DC voltage [29-31]. To improve performance under various loading conditions, references $[12,32,33]$ have proposed an adaptive scheme that modified the droop gains depending on the system state.

\subsection{Ratio control}

This configuration can be seen as a droop control scheme with additional parameters that provides more flexibility in the steering of the power flow [7, 34]. As in droop control, some stations work in constant power mode and other terminals regulate the DC voltage using the law

$$
I_{j}-I_{j}^{*}=n_{j} K_{I j}\left(U_{j}-U_{j}^{*}\right),
$$

where $n_{j}$ is the contribution part of each terminal with $\sum_{j} n_{j}=1$. It can be proved that the factors $n_{j}$ 's impose the power contribution ratio of each station regulating the voltage [34]. Therefore, by adjusting these parameters, the system operator can control the power participation of each terminal in the DC voltage regulation. The ratios can also be changed during operation, if communications are available, to adapt the power flow according to the generation conditions. 
However, the inclusion of communications may be a drawback as it increases costs and reduces reliability [34]. Another disadvantage of ratio control is that the computation of the parameters (ratios) for a desired power share can be cumbersome in case of a large number of stations [34].

\subsection{Priority control}

This scheme combines droop control and constant voltage modes, and is also known as master-slave with droop control or dead-band droop control. The idea is to give priority to one station over the rest in the power regulation. The station with high priority operates in constant voltage and the others in droop control [34-37]. The idea is illustrated in Figure 15 for a four-terminal network. Terminal 1 is the station with high priority and works in constant voltage control. Terminal 2 operates in constant power control as the rest of the terminals, but it is equipped with a droop control in case of abnormal conditions. In normal operation (point A in Figure 15), the DC voltage is regulated by the high priority station with a PI control law. In case of a fault occurs or the converter reaches its power limits or the value decided by the system operator, this station enters constant power mode. An increase in the voltage is caused by a momentary imbalance, which activates the droop controls of the other terminals. Under this circumstance, these stations take over the regulation of DC voltage. Figure 15 illustrates the scenario when a voltage fault occurs in the AC grid connected to the high priority terminal. This reduces the power limits, forcing the converter to enter constant power mode. As a result, the DC voltage rises and terminal 2 takes over the voltage regulation leading the system to a new equilibrium corresponding to point B in Figure 15.

Clearly, this scheme is useful when one of the terminals has precedence to inject power into the grid. One example is the islanded DC microgrid with generation and storage analysed in [38], where the terminal connected to the $\mathrm{AC}$ grid has precedence during normal operation. In case of a fault in the $\mathrm{AC}$ grid, the droop control in the other terminals is responsible for ensuring the power balance.

Priority control seems suitable for grids with a low number of terminals since the design and coordination of this scheme with a large number of stations could be cumbersome. Besides, by assigning priority to some terminals over others could result in a less effective use of the network capacity.

\subsection{Undead-band droop control}

Undead-band droop control is proposed in [39] and consists in all stations regulating the DC voltages configured with a modified droop control. Figure 16 illustrates the idea for a four-terminal network with two stations regulating the DC voltage.

The modified droop control consists of two regions with different droop gains. One droop slope corresponds to normal operation (point A in Figure 16) and the other to disturbed conditions (point B). When the DC voltage leaves the normal operating range, the strategy switches to a lower droop gain in order to ensure a tighter voltage regulation. A similar idea is proposed in [40]. 

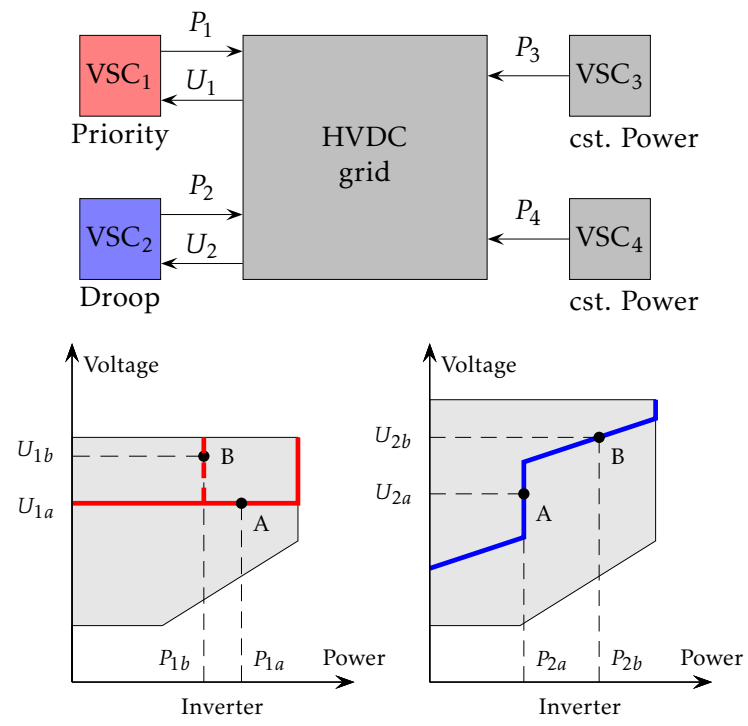

Figure 15: Example of a priority control strategy. In the upper sketch, the arrows represent information flow

This scheme offers, in addition to the same advantages than conventional droop control, an improved power flow regulation due to their different droop regions prepared for normal and fault conditions. The main drawback of undeadband droop control schemes is the need of high ratings for all power converters in order to ensure a wide operation range. Moreover, their design and extension to larger systems are difficult.

\subsection{Comparison of the different power sharing control schemes}

Table 1: Summary of some of the most relevant characteristics of the different control schemes for power sharing control in MT-HVDC networks

\begin{tabular}{|c|c|c|c|c|c|c|}
\hline $\begin{array}{l}\text { Control configu- } \\
\text { ration }\end{array}$ & Reliability & $\begin{array}{l}\text { Power } \\
\text { flow } \\
\text { steering }\end{array}$ & $\begin{array}{l}\text { VSC } \\
\text { rating }\end{array}$ & $\begin{array}{l}\text { Design } \\
\text { com- } \\
\text { plexity }\end{array}$ & Scalability & $\begin{array}{l}\text { Response } \\
\text { time }\end{array}$ \\
\hline Master-slave & low & high & high & low & high & low \\
\hline Margin voltage & medium & high & high & high & medium & high \\
\hline Priority & medium & medium & medium & high & medium & low \\
\hline $\begin{array}{l}\text { Undead-band } \\
\text { droop }\end{array}$ & high & medium & low & medium & medium & low \\
\hline Ratio & high & medium & low & high & low & low \\
\hline Droop & high & low & low & low & high & low \\
\hline
\end{tabular}

Table 1 gathers several features of the previously described control schemes for power sharing. The table allows comparing the schemes in terms of reliability, capability of steering power flow, demanded power rating to the VSC for 

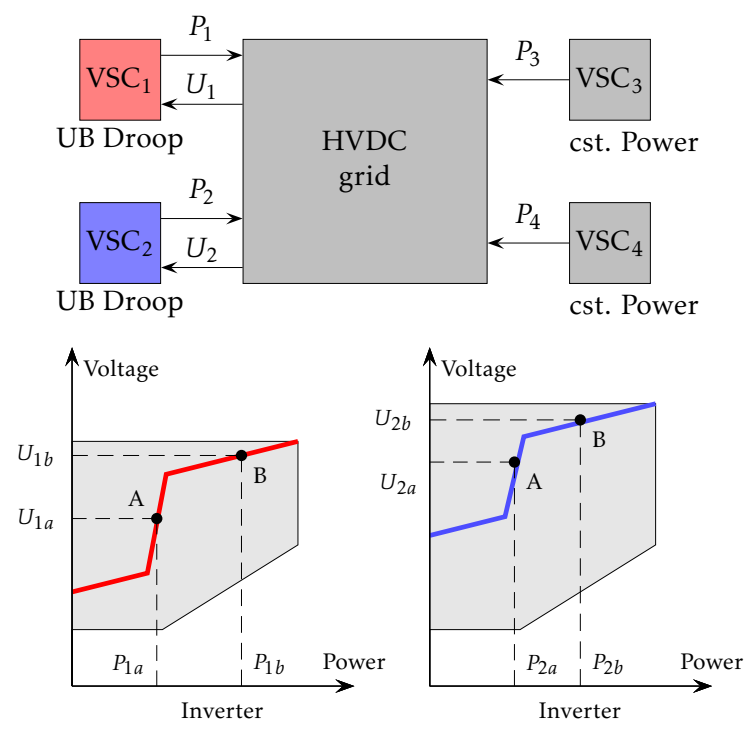

Figure 16: Example of an undead-band droop control strategy. In the upper sketch, the arrows represent information flow

voltage regulation, complexity, scalability and time response. It can be observed that control schemes like master-slave, with centralised authority, are capable of achieving a better power steering, but are less reliable. On the other hand, schemes with decentralised authority, such as droop control, are more reliable since several stations are responsible for regulating the voltage. However, it is more difficult to ensure an accurate power flow steering, although this can be corrected by the upper control levels. These conclusions are graphically summarised in Figure 17. Table 1 also shows that schemes with more centralised authority required higher power rating in the control terminals. Besides, improvements on master-slave schemes like margin voltage control increases reliability and reduces the VSC power rating, but at expenses of higher complexity reducing the scalability (i.e. the use in systems with a large number of stations) and increasing response times.

\section{Ancillary service provision from MT-HVDC networks}

As a consequence of the extensive use of power electronics in the current and future power transmission systems, new grid codes are under study. These codes impose strong requirements on power electronics based systems in order to allow their connection to the power systems. The fulfilment of these new requirements has encouraged the development of new control strategies for HVDC grids in the last years.

A large number of articles have proposed different control alternatives in order to use DC-links (two-terminal networks) for providing ancillary services to 


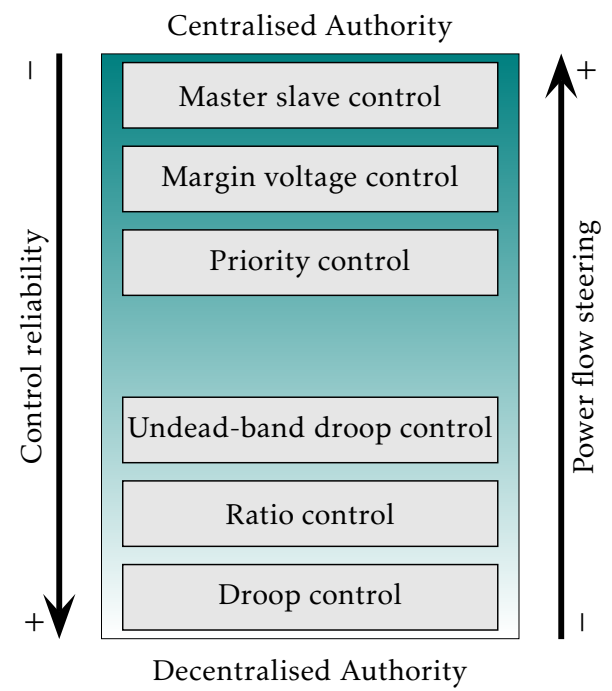

Figure 17: Graphic summary of the main features of different control schemes for power sharing in MT-HVDC networks

the AC grids, such as frequency support, damping of power oscillations, inertia emulation, and so on. However, a lower number of articles have considered the use of the additional degrees of freedom available for this purpose in MTHVDC networks. This is a consequence of the complex interactions among a great number of stations and the local controls, which makes difficult to extend DC-links ideas to multi-terminal networks.

This subsection analyses three main ancillary services (frequency support, inertia emulation and power oscillation damping) that could be potentially provided by the MT-HVDC networks.

\subsection{Frequency support}

In order to ensure a stable operation of AC systems, there must be a power balance between generation and demand. Any power imbalance is reflected as a fluctuation in the electrical frequency. For instance, a fully or partially loss of a generation unit causes a frequency fall governed by the total system inertia. Grid codes establish that generation plants must contribute to the frequency stability by regulating the active power injected into the grid. The grid codes also indicate the acceptable frequency response in case of power disturbances and classify the responses according to time ranges as: primary, secondary and tertiary responses. The primary response corresponds to the suitable frequency behaviour in the first seconds after the disturbance and establishes the maximum deviation and time ranges to stop the frequency decrease/increase. The secondary and tertiary responses concern with the restoration of the frequency to rated values and is normally provided by slow varying power reserves. Figure 18 shows a typical primary response established in the grid codes. The 


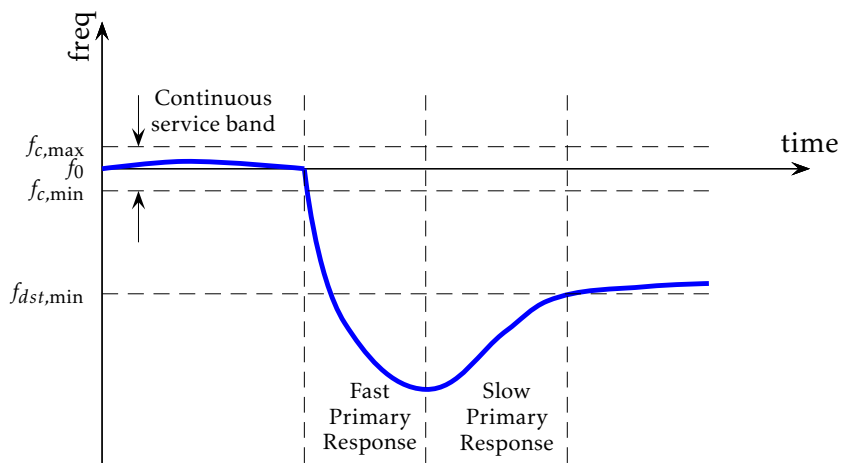

Figure 18: Typical expected primary frequency response, according to grid codes, where $f_{d s t, \text { min }}$ is the minimum steady state frequency, $f_{c, \text { min }}$ and $f_{c, \text { max }}$ are the limits of the frequency dead-band for the activation of primary reserves

primary response is in turn divided into two time ranges: fast and slow primary responses. The former is governed by the mechanical inertia (or fast response) of the generation units, and the latter by a control-loop aimed at stopping the frequency fall. Power electronics based systems, as MT-HVDC networks, are expected to contribute mainly to the primary frequency response. This section focuses on slow primary response, whilst inertia emulation (or fast primary response) is described later in Subsection 5.2.

Several researchers have tried to exploit the rapid response of VSCs and the distributed power reserves available in MT-HVDC grids to propose control strategies to provide primary frequency support in AC networks. These controls operate at the secondary level in the hierarchy scheme discussed in Section 2. The aim is to produce the suitable power set-points in order to reach a balance and lead the frequencies of the $\mathrm{AC}$ grid converter to a new equilibrium point. This allows sharing the power reserve and reducing costs. The first works were focussed on frequency control of two AC grids connected by HVDC links or tie-lines. In [41], the frequency stability of a four areas interconnected system with a DC tie-line in parallel to an AC line was analysed. Sanpei et al. [42] propose a centralised optimal control for two AC grids connected by a DC-link with the objective of maintaining the power balance. In [43, 44], it is analysed the capabilities of these systems to reduce frequency deviations after power disturbances, finding that when the AC grids are only connected by DC-links the contributions to the frequency regulation can be significant.

To extend previous results to multi-terminal networks can be cumbersome in case of a large number of terminals. In this case, the control must coordinate the contributions of all the stations and guarantee a stable and proper operation of the entire MT-HVDC grid. For these reasons, the research has been moved to distributed control concepts. For instance, Dai et al. [45] present a distributed control scheme to provide primary frequency support in non-synchronous AC networks connected by MT-HVDC grids. Further improvement to this scheme 
is presented in [46], in which cooperative control tools are used to enforce the frequencies of the $\mathrm{AC}$ grids to converge to a common value. Two control schemes are proposed. The first one uses the power injected by $N-1$ converters as control variables and a consensus algorithm (using the measures of the other terminals) to force a common frequency in all AC areas. The second scheme controls the DC voltage to reach a power balance and thus achieving a frequency regulation in all AC areas. This is a decentralised strategy that relies only on local measures. With a similar objective, Andreasson et al. [47] propose a decentralised control based on passivity concepts to ensure asymptotic stability. In all these cases, MT-HVDC networks are employed to stabilise the frequency in the AC grids by sharing active power among all stations.

\subsection{Inertia emulation}

According to ENTSO-E, synthetic or emulated inertia is "a facility provided by a power park module to replicate the effect of inertia of a synchronous power generating module to a prescribed level of performance". In other words, the concept refers to the generation unit capability to provide the fictitious inertia for network frequency stability support. ENTSO-E grid codes not only requests this special capability to generation units, but also to HVDC-links and DCconnected power park modules (which includes MT-HVDC networks). This can be understood as the capability to contribute to fast primary frequency response, the first seconds after the fault occurrence (frequency sags). Before reviewing the available proposal on the topic, a brief background on inertia emulation by using MT-HVDC grids is presented.

\subsubsection{Background on synthetic inertia emulation from MT-HVDC}

In $\mathrm{AC}$ systems, the system inertia $J$ defines the energy stored in the rotating masses of the synchronous generators, which may be used for fast-primary frequency response. The inertia of the $\mathrm{AC}$ network has critical influence on the frequency response as it governs the rate of change of frequency (ROCOF) according to

$$
\frac{2}{f_{0}} H \frac{d f}{d t}=P_{g}-P_{l}=\Delta P_{1}
$$

where $f_{0}$ is the nominal frequency, $H=J \omega^{2} / 2 S, P_{g}$ and $P_{l}$ represent the generation and consumption powers, respectively, and $\Delta P_{1}$ is the energy stored or delivered by the system inertia.

In DC networks, there is no rotating masses, but the energy stored in the DCside capacitors may play a role analogous to the system inertia $J$. Besides, as any power imbalance among stations is reflected in the DC voltage $U$, this variable can be considered as the analogous to the frequency $f$. Using these analogies, the dynamics of the $\mathrm{AC} / \mathrm{DC}$ power sharing can be stated in an expression similar to (3),

$$
\frac{N U}{S_{v s c}} C_{t o t} \frac{d U}{d t}=P_{\text {in }}-P_{\text {out }}=\Delta P_{2},
$$


where $N$ is the total number of capacitors in the multi-terminal system, $C_{t o t}$ is the sum of the DC-side capacitances and the average value of the line per each VSC converter, $S_{v s c}$ represents the rating of the VSC converter in VA, $P_{i n}$ and $P_{\text {out }}$ represent the input and output powers flowing through the converter, respectively, and $\Delta P_{2}$ is the energy stored or delivered by the system DC-side capacitance.

Comparing (3) and (4), it can be seen that the main difference between $\mathrm{AC}$ and DC networks is the time scale of the dynamic responses, seconds in $\mathrm{AC}$ systems and milliseconds in DC systems. In case of DC systems, the system inertia is governed by the total capacitance instead of the mechanical inertia in AC systems.

By comparing the energy stored in AC systems (3) and DC systems (4), the following expression can be obtained

$$
\frac{N U}{S_{v s c}} C_{t o t} \frac{d U}{d t}=\frac{2}{f_{0}} H_{v s c} \frac{d f}{d t} .
$$

After integrating both sides of (5) and simplifying, it results in

$$
\frac{2 H_{v s c} f}{f_{0}}=\frac{N C_{t o t} U^{2}}{2 S_{v s c}}+\underbrace{\left(2 H_{v s c}-\frac{N C_{t o t} U_{0}^{2}}{2 S_{v s c}}\right)}_{K_{0}} .
$$

Then, from (6), an emulated inertia can be computed as

$$
H_{v s c}=\frac{N C_{t o t} U_{0}^{2} f_{0}}{4 S_{v s c} \Delta f}\left(\left(\frac{\Delta U}{U_{0}}+1\right)^{2}-1\right) .
$$

The value $H_{v s c}$ indicates the equivalent inertia that a DC network with VSC is able to emulate.

\subsubsection{Control schemes for inertia emulation provision}

The main goal of synthetic inertia controls is to emulate the real inertia provided by synchronous generators. The emulation is obtained with the energy contribution of non-mechanical systems, such as VSCs, using a suitable control strategy. Two inertia emulation schemes based on VSC have been proposed in literature. Both schemes use the energy available in the DC capacitors to mitigate frequency sags and are applied to the grid-side converters of MT-HVDC networks. Despite the inertia emulation can be applied to any VSC of the multiterminal network, only one should be active at the same time in order to avoid interactions among converters that may result in negative effects.

The first scheme employs the frequency derivative measured at the grid connection point and varies the current set-points in order to regulate the active power contribution coming from the MT-HVDC grid [48]. In this case, the active power set-point is controlled by the variation of the $d$-axis current, as shown in Figure 19. The measure of the frequency derivative can be obtained from a 


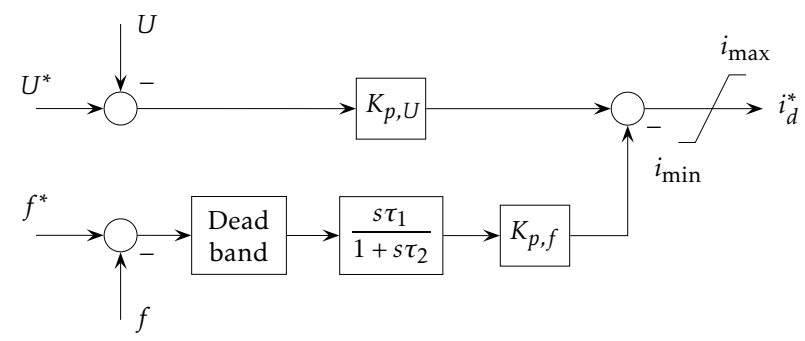

Figure 19: Synthetic inertia using frequency derivative control scheme

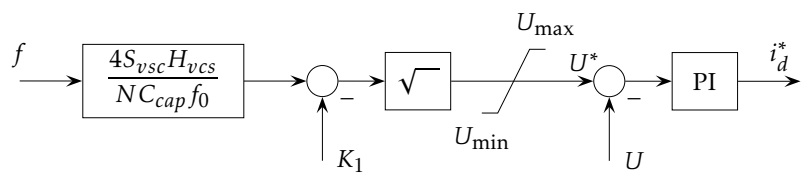

Figure 20: Synthetic inertia using INEC control scheme

washout filter with the aim of reducing the negative effect of noise. The scheme also includes a dead-band to prevent the control from acting during normal small frequency deviations.

The second synthetic inertia emulation controller (INEC) proposed in [48] measures the frequency with a phase locked loop (PLL) and acts on the DC voltage set-points of the power converters. These set-points are derived from (6) [49] and can be computed as

$$
U^{*}=\sqrt{\frac{4 S_{v s c} H_{v s c}}{N C_{t o t} f_{0}} f-K_{1}},
$$

where

$$
K_{1}=K_{0} 2 S_{v s c} / N C_{t o t}=4 S_{v s c} H_{v s c} / N C_{t o t}-U_{0}^{*} .
$$

The DC voltage set-point must remain within the maximum and minimum admissible converter limits. This scheme may result more reliable as it does not need the measure of the frequency derivative.

These previous schemes show that the use of the energy stored in the MTHVDC network capacitors can be used to effectively emulate the inertia of synchronous generators. The challenge is to improve stability margins and performance by coordinating the action of several stations.

\subsection{Power Oscillation Damping}

Power system oscillations appear as a result of excitation of natural frequencies associated with the equivalent system inertia and synchronous generators. In general, the damping of these power oscillations may be achieved by regulating both active and reactive powers [50]. 
The inclusion of power electronics within the power systems has added new controllable units that could be used to improve power system stability. The VSCs at the terminals of the HVDC networks are capable of providing fast contributions of active and reactive powers. Therefore, some articles have proposed to use these converters to help in the damping of power oscillations in AC systems. These schemes can be classified by the topology of HVDC networks in point-to-point HVDC-links and MT-HVDC grids.

\subsubsection{HVDC-links}

There are a number of results focussed on point-to-point HVDC-links. Although this review is focussed on MT-HVDC grids, previous experiences obtained from HVDC-links can give clues for potential control schemes. For instance, Venkataraman et al. [51] use $H_{\infty}$ optimal control with the aim of increasing the damping of the power systems using uncertainty representations to cover the dynamic changes associated with different operating points. In [52], exact linearisation is used with similar objectives. Hazra et al. [53] analyse the selection of several measures and control signals in order to achieve the maximum effect on the transient response of the AC grids. Pipelzadeh et al. [54] determine the most suitable control signals for the HVDC links to damp power oscillations, showing the potential of using active and reactive power simultaneously. In [55], a robust decentralised control is proposed using a homotopy approach to add various PSS active and reactive power loops with different input signals, improving reliability and robustness.

\subsubsection{Multi-terminal HVDC networks}

Providing oscillation damping from MT-HVDC grids results more complex as the coordination of several VSC controls is necessary. In multi-terminal networks, at least one converter must be responsible for regulating the DC voltage. The other converters can be used to control the power injected with the aim of damping power oscillations.

Ndreko et al. [56] propose three different control schemes (PSS-type, proportional and band-pass controls) acting on the active power, which exhibit promising capability for damping power oscillations in the AC grids. In [57], a design procedure is proposed to minimise the effect of abrupt DC voltage changes caused by converter disconnections. The procedure uses singular value decomposition (SVD) to determine the input direction with fewer effects on the AC grid side and thus computing the most suitable droop gains. On the other hand, Lyapunov stability theory is used to propose a nonlinear control law in order to ensure asymptotic stability in power systems including MT-HVDC networks [58]. Eriksson [59] presents an optimal droop scheme for voltage regulation in order to maximise controllability without using communications and to provide oscillation damping from MT-HVDC.

Most of these solutions are mainly focussed on active power regulation at the AC side of power converters. However, there are still some challenges to be faced such as the control design to ensure DC voltage stability and the selection of input and output signals to achieve the most effective damping. 


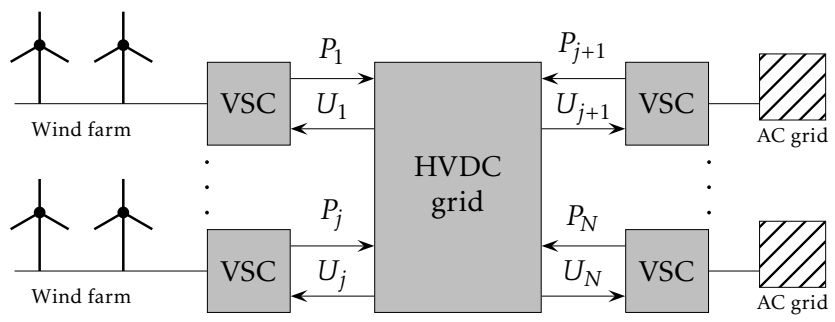

Figure 21: MT-HVDC grids in offshore wind power applications

\section{Control of MT-HVDC networks in wind power applications}

In case of offshore wind farms connected to onshore AC grids, the terminal stations corresponding to the wind farms commonly operate at constant power in order to deliver all power generated. The stations corresponding to the land grids can use several approaches in order to fulfil the objective of transferring the power generated at wind farms to consumers or loads in the land grids. Figure 21 sketches a MT-HVDC of $N$ stations for power sharing purposes, in which some of them are offshore wind farms.

When some of the terminals are WPPs, especially offshore wind farms, some of the concepts previously described about MT-HVDC networks must be rethought. The following particular features of WPPs should be considered to adapt the previously described control schemes.

- Power injection capability: WPPs are basically capable of injecting power into the MT-HVDC networks. Although some power extraction capability can be added with the help of energy storage systems or dissipation devices, the amount of power extracted from the grid is quite limited. This imposes serious constraints on the control strategies. In this case, the control must be mainly centred on the use of the AC grid terminals and must consider the limitations on the control variables at the WPP terminals.

- Reliability: As the main intention is to use the MT-HVDC networks to connect offshore WPPs, the reliability of the whole system is crucial. The maintenance of offshore facilities is considerably more expensive and more dependent on meteorological conditions than onshore installations. This implies that the control strategies must have fault tolerant capabilities and keep working even under certain abnormal conditions. Especially, the control scheme must have at least low-level control strategies to ensure a basic operation without communications.

- Highly variable power generation: One of the most significant difference of WPPs with respect to conventional power plants is the high variability of the power production. In case of MT-HVDC networks, this implies 
continuous changes in the power flow. As a consequence, the control schemes must be capable of properly working in different conditions.

- Free selection of electrical frequency: As there is no consume connected to WPPs, the electrical frequency in the wind farm AC grids can be freely selected. This provides an additional degree of freedom in the proposition of control strategies e.g. in cases of frequency support. In this case, the frequency in the WPPs can be adjusted using distributed control schemes like those proposed in [46].

The number of articles explicitly considering these distinctive features is considerably lower than those dealing with MT-HVDC grids with generic terminals. The most relevant results are presented in the following subsections.

\subsection{Control schemes for power sharing}

In order to ensure power sharing from wind farms to terrestrial grids, almost all schemes previously presented in Section 4 have been also adapted to multiterminal networks including WPPs.

\subsubsection{Master-slave}

The use of master-slave configuration in WPP applications is rather direct as one of the terrestrial areas must act as a master to regulate the DC voltage and ensure a proper power sharing. The terminals connecting wind power farms work in constant power mode injecting all the power generated. Considering the lack of reliability discussed in previous sections, this scheme is not common in wind power applications. A master-slave configuration is analysed in [60] for a four-terminal grid connecting three wind farms with a terrestrial grid, the work is more focussed on the implementation aspects and faulty conditions are not considered. In [61], it is addressed the optimisation of the power flow in MTHVDC with WPP with the aim of minimising transmission losses. Experimental results in a scaled test rig are presented in [7].

\subsubsection{Voltage margin control}

As in the previous scheme, the application of voltage margin control in WPP applications does not present significant differences with other applications. Some of the converters connecting the land AC grids are designed as master and backup stations. This configuration is not popular in the literature, only few results have analysed this scheme in case of terminals connected to WPPs. In [16], simulation results can be found with the aim of comparing with other configurations.

\subsubsection{Droop control}

Droop control seems to be the most popular strategy to regulate the DC voltage and to ensure the proper power sharing even under abnormal conditions. A configuration of droop and constant power is proposed in [62-64]. In normal 
operation, the grid side converter regulates the DC voltage with a current based droop law at each terminal,

$$
I-I^{*}=K_{I}\left(U-U^{*}\right) .
$$

The converters on the wind farm side operate at constant power configuration. In abnormal conditions, such as severe voltage faults in the $\mathrm{AC}$ grid, the power limits of one or more grid side converters can be reached reducing the capability to regulate the DC voltage. For these scenarios, wind farm converter equipped with droop control has been proposed in $[62,63]$. Figure 22 shows the strategy in the U-I plane in case of a two-terminal network. In normal operation, the system remains at point A with voltage $U_{1 a}$ at the grid side terminal and $U_{2 a}$ at the wind farm terminal. In case of faults in the $\mathrm{AC}$ grid side, the maximum power limits in the grid side converter decrease and cannot continue regulating the voltage. Now, the U-I characteristic of the grid side converter is given by the blue dashed line. As a consequence, the voltage rises forcing the wind farm converter to enter droop control mode. Thus, the equilibrium moves to point B.
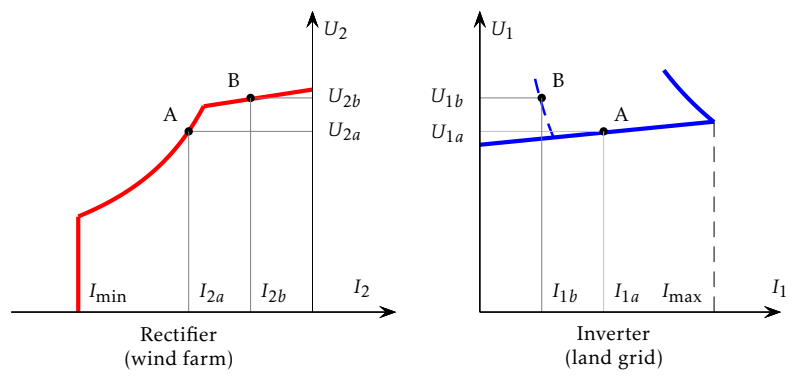

Figure 22: Droop control in WPP applications, dashed lines denote abnormal operation

Implementation aspects and detailed simulations are considered in [65, 66]. In the grid side converter, the implementation of droop control is more or less direct. However, the operation in abnormal conditions, in which the wind farm converters take over the DC voltage regulation, the implementation of the droop control is more complex. In [7,62], the droop control is implemented acting on the $\mathrm{AC}$ voltage with the aim of regulating the power injected by the wind farms in abnormal circumstances. In [67], three options for operating under faulty conditions are proposed: the use of communications to control the power generated, the modulation of the wind farm frequency, and the use of a DC damping resistor. Implementation aspects are further analysed in [26], in which the droop control in multi-terminal grids is studied in a scaled experimental platform where both normal and converter disconnections are studied.

\subsubsection{Ratio control}

Ratio control is applied in [7] in a scaled test rig including two grid-side converters and one wind-farm station. The ratio control is used to maintain 
constant the participation of both grid side converters in the voltage regulation. The application to a five-terminal network, with three land grids and two wind farms, is analysed in [68].

\subsubsection{Priority control}

Priority control is used, for instance, in [67], where one of the stations connected to the terrestrial grid works in constant voltage mode acting as master (terminal with higher priority) and the other grid side converters works in droop or ratio control. In this way, the wind power is conducted first to one particular $\mathrm{AC}$ grid and only when the wind farm production is too high the power excess is sent to the other $\mathrm{AC}$ grids.

\subsection{Control for ancillary services}

As in the applications analysed in Section 5, the multi-terminal networks in WPP applications will be probably required to contribute to system stability by providing ancillary services like frequency support, damping of power oscillations and inertia emulation. The provision of these services by WPPs has been extensively studied. However, the provision of these services by WPPs connected to AC land grid through MT-HVDC networks requires new control strategies. Since the wind farms are not directly connected to the main AC grids, the contribution will be provided by the grid-side converters of the MTHVDC networks (notice that the power may be also shared among different AC grids). In this circumstance and with the aim of not affecting other AC systems, ancillary services are expected to act on the active power delivered by the WPPs. For this purpose, wind farms may provide a power surplus in normal operation by slowing down the wind rotor (extracting the energy stored in the rotor) or by acting on the pitch control and the power converter to use power reserves in the wind turbines (deloading) [69-72].

The number of articles about the use WPP connected through MT-HVDC networks to provide ancillary services is not large. Silva et al. [73] propose a modified droop control in order to regulate the frequency in the AC grids by means of the DC voltage control. This scheme aims at providing fast and slow primary frequency response without communications. This is an important feature in MT-HVDC networks connecting offshore WPPs, as communications may result problematic in these applications [74]. In order to avoid interactions between the power sharing control presented in the previous works, Martinez Sanz et al. [75] propose a weighted frequency control strategy. The scheme is still a droop control scheme but uses communications to inform the offshore WPPs about the power contributions needed to compensate the frequency deviation in the $\mathrm{AC}$ grids. The simultaneous use of wind turbine inertia and the energy stored in the DC capacitors for providing fast primary frequency response is analysed in [76]. Although the work only considers HVDC-links transferring power from offshore WPPs, the proposed concepts could be also used in multiterminal networks.

Regarding power oscillation damping, Ndreko et al. [56] propose the implementation of an extra control loop that modifies the active power set-points of 
the wind farms with the aim of producing the necessary active power signal for damping power oscillations. In this case, communications among onshore AC grid converters (the ones which needs the contribution) and the offshore wind farm converters are required. In this scheme, the control of the MT-HVDC network does not participate in the service provision. A relevant issue to consider, especially for this ancillary service, is the potential interaction between the ancillary service control and the wind turbine mechanical structure, which may cause undesired torsional loads.

\section{Conclusions}

Power transmission systems based on MT-HVDC technologies are foreseen as one of the key elements in future power systems including offshore grids. As each station is equipped with electronic interfaces, the behaviour of MTHVDC networks is dominated by the control strategies. In general, the control in MT-HVDC grid involves several levels, each one with a particular goal.

The power sharing among stations is mainly governed by the primary control level. This level is, in general, a decentralised control scheme in order to ensure a proper operation even under communication faults. The control schemes proposed in the literature range from a highly centralised authority (master-slave) to more decentralised authority like droop control. The first schemes ensure a better power flow steering, but low reliability. The opposite occurs with the second group. In order to enhance the advantages of each group, several intermediate solutions have been proposed. The trend in the research favours the droop-type control, although in the first real world application a voltage margin control scheme has been chosen.

With the expected growth of MT-HVDC networks, and the consequent reduction of the power system inertia and the increase in the controllability, the provision of ancillary services demanded to MT-HVDC grids will increase. The main contributions of MT-HVDC grids will be in frequency support, power oscillation damping and inertia emulation. The frequency support schemes are in general based on distributed control ideas seeking to share active power among $\mathrm{AC}$ areas (no power reserve in $\mathrm{DC}$ grid) while maintaining the voltage of the HVDC grid stable. The potential use of the energy stored in DC grids to provide frequency support and thus reducing the power shared among $\mathrm{AC}$ areas may be worthy of being studied. The control strategies for power oscillation damping are focussed on the regulation of the active power in the AC side without significantly affecting the DC grid stability. The use of reactive power for damping oscillations, as proposed for other power electronic based technologies (e.g. FACTS or wind power), as well as the impact of power converter loading, has not been extensively analysed in the literature. Regarding inertia emulation, the proposed control schemes use the existing capacitors on the MT-HVDC networks to emulate the concept of inertia in the AC system. However, there is no study on system energy recovery or on its effects on the systems.

When some of the stations are WPPs, both the power sharing and the ancillary service provision schemes must be rethought. For power sharing purposes, 
wind power terminals are commonly injecting power into the DC grid with a specific power curve, which modifies the overall response of the system. In case of provision of ancillary services, the challenges are in minimising the use of communications and interactions with the power sharing control.

\section{Acknowledgements}

This work was supported by the EU $7^{\text {th }}$ framework programs FP7ENERGY-2013 IRPWIND Project (under Grant Agreement 609795).

[1] EWEA, . Eu energy policy to 2050: Achieving 80-95\% emission reductions. Tech Report.; European Wind Energy Association (EWEA); 2011. URL: http://www.ewea.org/fileadmin/files/library/publications/ reports/EWEA_EU_Energy_Policy_to_2050.pdf.

[2] EWEA, . The european wind initiative: Wind power research and development for the next ten years. Tech Report; European Wind Energy Association (EWEA); 2010. URL: http://www . ewea.org/fileadmin/ewea_ documents/documents/publications/EWI/EWI_2010_final.pdf.

[3] EWEA, . Wind in power: 2014 european statistics. Tech. Rep.; European Wind Energy Association (EWEA); 2015. URL: http://www.ewea.org/fileadmin/files/library/publications/ statistics/EWEA-Annual-Statistics-2014.pdf.

[4] Gordon, S.. SuperGrid to the rescue. IET Power Eng 2006;20(5):30-33.

[5] Van Hertem, D., Ghandhari, M.. Multi-terminal VSC HVDC for the European supergrid: Obstacles. Renewable Sustainable Energy Rev 2010;14:3156-3163.

[6] Hao, W., Luo, L., Diafeng, W., Pudney, D.. Hierarchical control in a 5-terminal VSC-HVDC project. Energize 2014;83.

[7] Liang, J., Jing, T., Gomis-Bellmunt, O., Ekanayake, J., Jenkins, N.. Operation and control of multiterminal HVDC transmission for offshore wind farms. IEEE Trans Power Delivery 2011;26(4):2596-2604. doi:10. 1109/TPWRD . 2011.2152864.

[8] ENTSO-E, . Draft network code on high voltage direct current connection and DC-connected power park modules. 2014.

[9] Egea-Alvarez, A., Beerten, J., Van Hertem, D., Gomis-Bellmunt, O.. Primary and secondary power control of multiterminal HVDC grids. In: Proc. of 10th IET International Conference on AC and DC Power Transmission (ACDC). 2012,doi:10.1049/cp. 2012.1989. 
[10] Teixeira Pinto, R., Bauer, P., Rodrigues, S.F., Wiggelinkhuizen, E.J., Pierik, J., Ferreira, B.. A novel distributed direct-voltage control strategy for grid integration of offshore wind energy systems through MTDC network. IEEE Trans Ind Electron 2013;60(6):2429-2441. doi:10.1109/tie . 2012.2216239.

[11] Teixeira Pinto, R., Rodrigues, S., Wiggelinkhuizen, E.J., Scherrer, R., Bauer, P., Pierik, J.. Operation and power flow control of multi-terminal dc networks for grid integration of offshore wind farms using genetic algorithms. Energies 2013;6:1-26.

[12] Khazaei, J., Miao, Z., Piyasinghe, L., Fan, L.. Minimizing DC system loss in multi-terminal HVDC systems throughadaptive droop control. Electr Pow Syst Res 2015;126:78-86. doi:10.1016/j.epsr.2015.04.020.

[13] Aragüés-Peñalba, M., Egea-Alvarez, A., Arellano, S.G., Gomis-Bellmunt, O.. Droop control for loss minimization in HVDC multi-terminal transmission systems for large offshore wind farms. Electr Pow Syst Res 2014;112:48-55. doi:10.1016/j.epsr.2014.03.013.

[14] Ekanayake, J.. Multi-terminal DC converters for connecting induction generator based distribution generation. In: Proc. of the International Conference on Industrial and Information Systems (ICIIS). 2009, p. 466471. doi:10.1109/ICIINFS. 2009.5429815.

[15] Zhang, L., Wang, Y., Li, H.. Coordinated control of MTDC-based microgrid with wind turbines. In: Proc. of the 7th International Power Electronics and Motion Control Conference (IPEMC); vol. 3. 2012, p. 20762080. doi:10.1109/IPEMC . 2012.6259163.

[16] Teixeira Pinto, R., Rodrigues, S., Bauer, P., Pierik, J.. Comparison of direct voltage control methods of multi-terminal DC (MTDC) networks through modular dynamic models. In: Proc. of the 14th European Conference on Power Electronics and Applications (EPE). 2011, p. 1-10.

[17] Tokiwa, Y., Ichikawa, F., Syzuki, K., Inokuchi, H., Hirose, S., Kimura, K.. Novel control strategies for HVDC system with self-contained converter. Electrical Engineering in Japan (English translation of Denki Gakkai Ronbunshi) 1993;113(5):1-13.

[18] Nakajima, T., Irokawa, S.. A control system for HVDC transmission by voltage sourced converters. In: Proc. of the IEEE Power Engineering Society Summer Meeting Conference; vol. 2. 1999, p. 1113-1119. doi:10. 1109/PESS. 1999.787474.

[19] Zhu, J., Booth, C.. Future multi-terminal HVDC transmission systems using voltage source converters. In: Proc. of the 45th International Power Engineering Conference (UPEC). 2010, p. 1-6. 
[20] Mier, V., Casielles, P., Coto, J.D., Zeni, L.. Voltage margin control for offshore multi-use platform integration. In: Proc. of the European Association for the Development of Renewable Energies, Environment and Power Quality (EA4EPQ). 2012,.

[21] Teixeira Pinto, R., Rodrigues, S., Bauer, P., Pierik, J.. Description and comparison of DC voltage control strategies for offshore MTDC networks: Steady-state and fault analysis. EPE Journal 2012;22(4):31-39.

[22] Beerten, J., Cole, S., Belmans, R.. Modeling of multi-terminal VSC HVDC systems with distributed DC voltage control. IEEE Trans Power Syst 2014;29(1):34-42. doi:10.1109/tpwrs . 2013.2279268.

[23] Teixeira Pinto, R., Rodrigues, S., Bauer, P., Pierik, J.. Multi-terminal DC networks. Ph.D. thesis; Delft University of Technology; 2013.

[24] Johnson, B., Lasseter, R., Alvarado, F., Adapa, R.. Expandable multiterminal dc systems based on voltage droop. IEEE Trans Power Delivery 1993;8(4):1926-1932. doi:10.1109/61.248304.

[25] Beerten, J., Belmans, R.. A comprehensive modeling framework for dynamic and steady-state analysis of voltage droop control strategies in HVDC grids. Int J Electr Power Energy Syst 2016;73:691-701. doi:10. 1016/j.ijepes.2015.05.030.

[26] Egea-Alvarez, A., Bianchi, F., Junyent-Ferre, A., Gross, G., GomisBellmunt, O.. Voltage control of multiterminal VSC-HVDC transmission systems for offshore wind power plants: Design and implementation in a scaled platform. IEEE Trans Ind Electron 2013;60(6):2381-2391.

[27] Haileselassie, T., Uhlen, K.. Impact of DC line voltage drops on power flow of MTDC using droop control. IEEE Trans Power Syst 2012;27(3):14411449. doi:10.1109/TPWRS. 2012.2186988.

[28] Hendriks, R., van der Meer, A., Kling, W.. Impact on system stability of different voltage control schemes of wind power plants connected through $\mathrm{AC}$ and VSC-HVDC transmission. In: Proc. of the Nordic Wind Power Conference. Rnne, Denmark; 2009, p. 1-8.

[29] Prieto-Araujo, E., Bianchi, F., Junyent-Ferre, A., Gomis-Bellmunt, O.. Methodology for droop control dynamic analysis of multiterminal VSC-HVDC grids for offshore wind farms. IEEE Trans Power Delivery 2011;26(4):2476-2485. doi:10.1109/TPWRD. 2011.2144625.

[30] Kalcon, G.O., Adam, G.P., Anaya-Lara, O., Lo, S., Uhlen, K.. Smallsignal stability analysis of multi-terminal vsc-based DC transmission systems. IEEE Trans Power Syst 2012;27(4):1818-1830. doi:10.1109/tpwrs . 2012.2190531. 
[31] Gavriluta, C., Candela, I., Citro, C., Luna, A., Rodriguez, P.. Design considerations for primary control in multi-terminal VSC-HVDC grids. Electr Pow Syst Res 2015;122:33-41. doi:10.1016/j.epsr.2014.12.020.

[32] Chaudhuri, N.R., Chaudhuri, B.. Adaptive droop control for effective power sharing in multi-terminal DC (MTDc) grids. IEEE Trans Power Syst 2013;28(1):21-29. doi:10.1109/tpwrs . 2012.2203390.

[33] Ran, X., Miao, S., Wu, Y.. Improved adaptive droop control design for optimal power sharing in VSC-MTDC integrating wind farms. Energies 2015;8:7100-7121. doi:10.3390/en8077100.

[34] Xu, L., Yao, L., Bazargan, M.. DC grid management of a multi-terminal HVDC transmission system for large offshore wind farms. In: Proc. of the International Conference on Sustainable Power Generation and Supply (SUPERGEN '09). 2009, p. 1-7. doi:10.1109/SUPERGEN . 2009.5348101.

[35] Rodrigues, S., Teixeira Pinto, R., Bauer, P.. Dynamic Modeling and Control of VSC-based Multi-terminal DC Networks: With focus on offshore wind energy systems.; vol. 1. Lambert Academic Publishing; 2012.

[36] Dierckxsens, C., Srivastava, K., Reza, M., Cole, S., Beerten, J., Belmans, R.. A distributed DC voltage control method for VSC MTDC systems. Electr Power Syst Res 2012;82(1):54-58. doi:10.1016/j.epsr.2011.08. 006.

[37] Vrana, T.K., Beerten, J., Belmans, R., Fosso, O.B.. A classification of DC node voltage control methods for HVDC grids. Electr Power Syst Res 2013;103:137-144. doi:10.1016/j .epsr.2013.05.001.

[38] Chen, D., Xu, L.. Autonomous DC voltage control of a DC microgrid with multiple slack terminals. IEEE Trans Power Syst 2012;27(4):18971905. doi:10.1109/TPWRS . 2012.2189441.

[39] Vrana, T., Zeni, L., Fosso, O.. Dynamic active power control with improved undead-band droop for HVDC grids. In: Proc. of the 10th International Conference on AC and DC Power Transmission. 2012,.

[40] Barker, C., Whitehouse, R.. Further developments in autonomous converter control in a multi-terminal HVDC system. In: Proc. of 10th IET International Conference on AC and DC Power Transmission (ACDC). 2012,doi:10.1049/cp.2012.1976.

[41] Bhamidipati, S., Kumar, A.. Load frequency control of an inter-connected system with DC tie-lines and AC-DC parallel tie-lines. In: Proc. of the Twenty-Second Annual North American Power Symposium. 1990, p. 390395. doi:10.1109/NAPS . 1990.151393. 
[42] Sanpei, M., Kakehi, A., Takeda, H.. Application of multi-variable control for automatic frequency controller of HVDC transmission system. IEEE Trans Power Delivery 1994;9(2):1063-1068. doi:10.1109/61.296291.

[43] Fujita, G., Shirai, G., Yokoyama, R.. Automatic generation control for DC-link power system. In: Proc. of the Asia Pacific IEEE/PES Transmission and Distribution Conference and Exhibition; vol. 3. 2002, p. 1584-1588 vol.3. doi:10.1109/TDC. 2002.1176835.

[44] Sterpu, S., Tuan, M.. Sharing frequency response between asynchronous electrical systems. In: Proc. of the Power Energy Society General Meeting. 2009, p. 1-6. doi:10.1109/PES.2009.5275627.

[45] Dai, J., Phulpin, Y., Sarlette, A., Ernst, D.. Coordinated primary frequency control among non-synchronous systems connected by a multiterminal high-voltage direct current grid. IET Gener Transm Distrib 2012;6(2):99. doi:10.1049/iet-gtd.2011.0312.

[46] Sarlette, A., Dai, J., Phulpin, Y., Ernst, D.. Cooperative frequency control with a multi-terminal high-voltage DC network. Automatica 2012;48(12):3128-3134. doi:10.1016/j . automatica.2012.08.017.

[47] Andreasson, M., Wiget, R., Dimarogonas, D.V., Johansson, K.H., Andersson, G.. Distributed primary frequency control through multi-terminal HVDC transmission systems. arXiv:1409.8013; 2014. arXiv:1409.8013.

[48] Bucurenciu, A.M.. Primary Frequency Response by MTDC Offshore Grids. Master's thesis; Delft University of Technology; 2012.

[49] Zhu, J., Guerrero, J., Hung, W., Booth, C., Adam, G.. Generic inertia emulation controller for multi-terminal voltage-source-converter high voltage direct current systems. IET Renew Power Gener 2014;8(7):740-748. doi:10.1049/iet-rpg. 2014.0109.

[50] Domínguez-García, J.L., Gomis-Bellmunt, O., Bianchi, F., Sumper, A.. Power oscillation damping supported by wind power: A review. Renewable Sustainable Energy Rev 2012;16(7):4994-5006. doi:10.1016/j.rser. 2012.03 .063$.

[51] Venkataraman, S., Khammash, M., Vittal, V.. Analysis and synthesis of HVDC controls for robust stability of power systems. IEEE Trans Power Syst 1995;10(4):1933-1938.

[52] Eriksson, R., Knazkins, V., Söder, L.. Coordinated control of multiple HVDC links using inputoutput exact linearization. Electr Power Syst Res 2010;80(12):1406-1412. doi:10.1016/j.epsr.2010.06.001.

[53] Hazra, J., Phulpin, Y., Ernst, D.. HVDC control strategies to improve transient stability in interconnected power systems. In: Proc. of the Bucharest PowerTech. 2009, p. 1-6. doi:10.1109/PTC.2009.5281816. 
[54] Pipelzadeh, Y., Chaudhuri, N., Chaudhuri, B., Green, T.. System stability improvement through optimal control allocation in voltage source converter-based high-voltage direct current links. IET Gener Transm Distrib 2012;6(10):811-821.

[55] Pipelzadeh, Y., Chaudhuri, B., Green, T.. Control coordination within a VSC HVDC link for power oscillation damping: A robust decentralized approach using homotopy. IEEE Trans Control Syst Technol 2013;21(4):12701279. doi:10.1109/TCST.2012.2202285.

[56] Ndreko, M., Van der Meer, A., Rawn, B., Gibescu, M.. Damping of power system oscillations by VSC-HVDC multi-terminal transmission networks. Project Technical Report, Part I; Delft University of Technology; 2013.

[57] Eriksson, R., Beerten, J., Ghandhari, M., Belmans, R.. Optimizing DC voltage droop settings for AC/DC system interactions. IEEE Trans Power Delivery 2014;29(1):362-369. doi:10.1109/TPWRD . 2013. 2264757.

[58] Eriksson, R.. On the centralized nonlinear control of HVDC systems using lyapunov theory. IEEE Trans Power Delivery 2013;28(2):1156-1163. doi:10.1109/TPWRD . 2013.2240021.

[59] Eriksson, R.. A new control structure for multi-terminal dc grids to damp inter-area oscillations. IEEE Trans Power Delivery 2014;:In press doi:10. 1109/TPWRD. 2014.2364738.

[60] Lu, W., Ooi, B.T.. Optimal acquisition and aggregation of offshore wind power by multiterminal voltage-source HVDC. IEEE Trans Power Delivery 2003;18(1):201-206. doi:10.1109/TPWRD . 2002.803826.

[61] Cao, J., Du, W., Wang, H.F., Bu, S.Q.. Minimization of transmission loss in meshed AC/DC grids with VSC-MTDC networks. IEEE Trans Power Syst 2013;28(3):3047-3055. doi:10.1109/tpwrs .2013. 2241086.

[62] Liang, J., Gomis-Bellmunt, O., Ekanayake, J., Jenkins, N.. Control of multi-terminal VSC-HVDC transmission for offshore wind power. In: Proc. of the 13th European Conference on Power Electronics and Applications (EPE 09). 2009,.

[63] Gomis-Bellmunt, O., Liang, J., Ekanayake, J., Jenkins, N.. Voltagecurrent characteristics of multiterminal HVDC-VSC for offshore wind farms. Electr Power Syst Res 2011;81(2):440-450. doi:10.1016/j .epsr . 2010.10. 007.

[64] Zhu, J., Booth, C.D., Adam, G., Roscoe, A.J.. Coordinated direct current matching control strategy formulti-terminal DC transmission systems with integrated wind farms. Electr Pow Syst Res 2015;124:55-64. doi:10.1016/j.epsr.2015.02.015. 
[65] Craciun, B.I., da Silva, R., Teodorescu, R., Rodriguez, P.. Multilink DC transmission for offshore wind power integration. In: Proc. of the IEEE International Symposium on Industrial Electronics. 2012, p. 1894-1899. doi:10.1109/ISIE. 2012.6237381.

[66] Egea-Alvarez, A.. Operation and control of VSC-HVDC multiterminal grids for offshore wind. In: Proc. of EPE. 2011,.

[67] Xu, L., Yao, L.. DC voltage control and power dispatch of a multi-terminal HVDC system for integrating large offshore wind farms. IET Renew Power Gener 2011;5(3):223. doi:10.1049/iet-rpg.2010.0118.

[68] Nieradzinska, K.. Generalised droop control for power management in a multi-terminal HVDC system. In: Proc. of EWEA. 2012,.

[69] Morren, J., de Haan, S., Kling, W., Ferreira, J.. Wind turbines emulating inertia and supporting primary frequency control. IEEE Trans Power Syst 2006;21(1):433-434. doi:10.1109/tpwrs . 2005.861956.

[70] de Almeida, R.G., Pecas Lopes, J.A.. Participation of doubly fed induction wind generators in system frequency regulation. IEEE Trans Power Syst 2007;22(3):944-950. doi:10.1109/tpwrs . 2007.901096.

[71] Anaya-Lara, O., Jenkins, N., Ekanayake, J., Cartwright, P., Hughes, M.. Wind Energy Generation: Modelling and Control. John Wiley \& Sons; 2009 .

[72] Díaz-González, F., Hau, M., Sumper, A., Gomis-Bellmunt, O.. Participation of wind power plants in system frequency control: Review of grid code requirements and control methods. Renewable Sustainable Energy Rev 2014;34:551-564. doi:10.1016/j.rser.2014.03.040.

[73] Silva, B., Moreira, C., Seca, L., Phulpin, Y., Peças-Lopez, J.. Provision of inertial and primary frequency control services using offshore multiterminal HVDC networks. IEEE Trans Sustainable Energy 2012;3(4):800808.

[74] Silva, B.. Multi-terminal HVDC grids: Control strategies for ancillary services provision in interconnected transmission systems with offshore wind farms. Ph.D. thesis; Universidade do Porto; 2013.

[75] Martinez Sanz, I., Chaudhuri, B., Strbac, G.. Inertial response from offshore wind farms connected through DC grids. IEEE Trans Power Syst 2014;:In press doi:10.1109/tpwrs.2014.2349739.

[76] Junyent Ferre, A., Pipelzadeh, Y., Green, T.C.. Blending HVDC-link energy storage and offshore wind turbine inertia for fast frequency response. IEEE Trans Sustainable Energy 2014;:In press doi:10.1109/tste.2014. 2360147. 